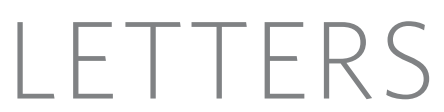

\title{
Environmental context explains Lévy and Brownian movement patterns of marine predators
}

\author{
Nicolas E. Humphries ${ }^{1,2}$, Nuno Queiroz ${ }^{1,3,4}$, Jennifer R. M. Dyer ${ }^{1}$, Nicolas G. Pade ${ }^{1,4}{ }^{\text {, Michael K. Musyl }}{ }^{5}$, \\ Kurt M. Schaefer ${ }^{6}$, Daniel W. Fuller ${ }^{6}$, Juerg M. Brunnschweiler ${ }^{7}$, Thomas K. Doyle ${ }^{8}$, Jonathan D. R. Houghton ${ }^{9}$, \\ Graeme C. Hays ${ }^{10}$, Catherine S. Jones ${ }^{4}$, Leslie R. Noble ${ }^{4}$, Victoria J. Wearmouth ${ }^{1}$, Emily J. Southall ${ }^{1}$ \\ \& David W. Sims ${ }^{1,2}$
}

\begin{abstract}
An optimal search theory, the so-called Lévy-flight foraging hypothesis ${ }^{1}$, predicts that predators should adopt search strategies known as Lévy flights where prey is sparse and distributed unpredictably, but that Brownian movement is sufficiently efficient for locating abundant prey ${ }^{2-4}$. Empirical studies have generated controversy because the accuracy of statistical methods that have been used to identify Lévy behaviour has recently been questioned ${ }^{5,6}$. Consequently, whether foragers exhibit Lévy flights in the wild remains unclear. Crucially, moreover, it has not been tested whether observed movement patterns across natural landscapes having different expected resource distributions conform to the theory's central predictions. Here we use maximum-likelihood methods to test for Lévy patterns in relation to environmental gradients in the largest animal movement data set assembled for this purpose. Strong support was found for Lévy search patterns across 14 species of open-ocean predatory fish (sharks, tuna, billfish and ocean sunfish), with some individuals switching between Lévy and Brownian movement as they traversed different habitat types. We tested the spatial occurrence of these two principal patterns and found Lévy behaviour to be associated with less productive waters (sparser prey) and Brownian movements to be associated with productive shelf or convergence-front habitats (abundant prey). These results are consistent with the Lévy-flight foraging hypothesis ${ }^{1,7}$, supporting the contention ${ }^{8,9}$ that organism search strategies naturally evolved in such a way that they exploit optimal Lévy patterns.
\end{abstract}

Lévy flights are a special class of random walk with movement displacements (steps) drawn from a probability distribution with a power-law tail (the so-called Pareto-Lévy distribution) ${ }^{1,10}$, and give rise to stochastic processes closely linked to fractal geometry and anomalous diffusion phenomena ${ }^{7,11}$. Lévy flights describe a movement pattern characterized by many small steps connected by longer relocations, with this pattern having scale invariance under projection, such that the probability density function, $P\left(l_{j}\right)$, has a power-law tail in the long-distance regime: $P\left(l_{j}\right) \approx l_{j}^{-\mu}$, where $l_{j}$ is the flight length (step length of move $j$ ), and $\mu, 1<\mu \leq 3$, is the power-law exponent. Lévy flights comprise instantaneous steps and hence involve infinite velocities, whereas a Lévy walk ${ }^{10}$ refers to a finitevelocity walk such that displacement is determined after a time $t$, reflecting a dynamical process such as movement ${ }^{1,10,11}$. Lévy flights and walks are theorized to be the most efficient movement pattern for locating patchy prey in low concentrations on spatial scales beyond a searcher's sensory range, with an optimal search having a power-law exponent of $\mu \approx 2$ (refs 4,13$)$. It is proposed that organisms have therefore naturally evolved search patterns that can be modelled as optimal Lévy flights ${ }^{1,7,13}$.

However, burgeoning empirical support for this hypothesis recently foundered following studies suggesting methodological shortcomings in the estimation of power-law exponents and in determining the goodness of fit to the data ${ }^{5,6,14-16}$, thus casting doubt on some, if not all, of the empirical studies that used such methods ${ }^{8,9}$. Hence, controversy remains over whether Lévy behaviour occurs in nature $^{6,9,17}$, despite many empirical studies ${ }^{1,18}$. Furthermore, long time series of movements (over weeks to months) derived from animalattached electronic tags will very probably capture complex movement data resulting from different types of behaviour (for example searching, travelling and resting) as animals respond to various biotic and abiotic factors over time. Previous studies analysing movement data $^{6,12,13}$ on free-ranging animals for Lévy motion used data collected over long time periods and different habitat types, without giving sufficient consideration to the issue of there being different types of behaviour interspersed within the time series. The lack of analysis of separate behaviour-pattern types may be at least one reason why evidence for Lévy flights in animal behaviour has proved challenging to detect unequivocally ${ }^{9,17}$.

Here we present an analysis of the largest data set of recorded movements ( $n=12,294,347$ steps) assembled to test the Lévy-flight foraging (LFF) hypothesis ${ }^{1}$ using statistical methods (maximumlikelihood estimation (MLE) and Akaike information criteria (AIC) weights for model comparisons) that are considered robust and accurate $^{6,14-16}$. To test the predictions of the LFF hypothesis, we focused our analysis on vertical movement data recorded over 5,700 days using electronic tags attached to open-ocean predators (sharks, tunas, billfish and ocean sunfish; Methods and Supplementary Table 1). These species may be among those most likely to exhibit Lévy behaviour because they occupy unpredictable and depauperate environments with highly patchy prey distributions ${ }^{13}$, where Lévy motion is proposed to increase new-patch encounter probability ${ }^{19}$.

To allow for a more robust test of the LFF hypothesis, long and complex time series of vertical diving movements (hereafter tracks, or sections) undertaken as fish moved horizontally across their

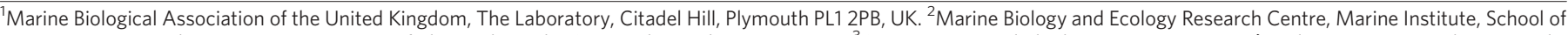

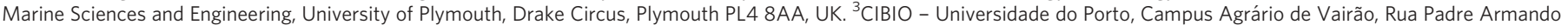

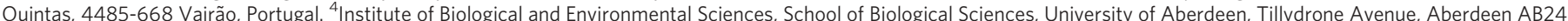

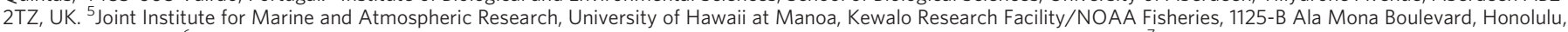

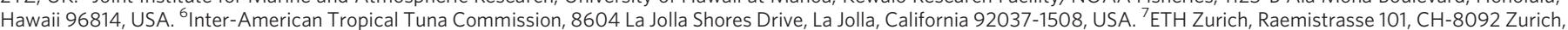

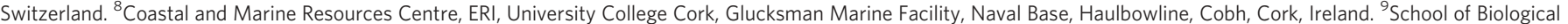
Sciences, Queen's University Belfast, Medical Biology Centre, 97 Lisburn Road, Belfast BT9 7BL, UK. ${ }^{10}$ Department of Pure and Applied Ecology, Institute of Environmental Sustainability, Swansea University, Singleton Park, Swansea SA2 8PP, UK.
} 
ranges were divided into shorter sections using a split movingwindow analysis ${ }^{20}$ (Supplementary Information, sections 1.2 and 1.3) to identify discontinuities in the pattern of vertical space use that represent transitions from one pattern of space use to another. In total, tracks from 55 individuals across 14 species (shark, eight species; tuna, two; billfish, three; ocean sunfish, one) were divided into 129 sections. MLE methods ${ }^{16}$ were used to fit three models (power law, truncated power law (truncated Pareto) or exponential) to the observed move step-length frequency distributions (Supplementary Information, section 1.4). Sections that from visual inspection proved to be poor fits to all candidate distributions were excluded from further analysis $(n=35)$, because our objective was to test the spatial occurrence of good fits to step-length distributions. MLE methods with AIC weights ${ }^{6,15}$ were then used to determine model best fits for the remaining 94 sections. Because movements can only take place in finite space (moves are limited by, for example, the sea surface, the sea bed or the range edge), which leads to upper cut-offs in the move step-length frequency distribution, only truncated Lévy walks are biologically plausible ${ }^{1}$. Therefore, our principal intention was not to find which kinds of all possible probability distributions best fit the data; rather, it was to test between truncated Lévy (truncated power-law model) and Brownian-type (exponential model) movement patterns.

We found clear and persistent signals of Lévy and Brownian motion; of the 94 sections analysed statistically (MLE with AIC weights), one section was best fitted by a pure power law (Fig. 1a-c) and 60 sections were best fitted by a truncated Pareto-Lévy distribution (see, for example, Fig. 1d-f and Supplementary Table 3 ) with exponents in the Lévy range, $1<\mu \leq 3$, and so were consistent with Lévy behaviour. The mean $\mu$ value for the Lévy sections was 1.94 (s.d., $0.43 ; n=61$ ), which is close to the proposed optimum, $\mu_{\mathrm{opt}} \approx 2$ (refs $1,2,4)$. Six sections best fitted by a truncated power law yielded exponents outside the Lévy range.

Lévy searching in open-ocean predators therefore seems to be not only present but prevalent; however, it does not seem to be a universal pattern, explaining all movements, nor does it occur in all individuals at all times (it occurs in only $47 \%$ of sections). A logical extension of the hypothesis is that other movement behaviour types intersperse Lévy patterns. In support of this, we found that 27 sections (21\%) were best fitted by an exponential model describing normal random processes (Brownian motion; Supplementary Table 3) that under the LFF hypothesis are consistent with optimal searches where prey is abundant ${ }^{1}$. We also found that 35 sections (27\%) were poorly fitted by all of the distributions; this was perhaps because the sections comprised many different movement patterns, making them too complex for the statistical methods used here (Supplementary Information, section 1.5).

To investigate the environmental context of different behaviour patterns, we mapped the horizontal tracks of individual predators in the Atlantic or the Pacific ocean to determine in which types of habitat the sections showing Lévy and Brownian vertical movement patterns occurred. For example, in productive waters of the equatorial convergence front of the central North Pacific, the entire track of a silky shark (Carcharhinus falciformis) was best fitted by an exponential model, whereas for another silky shark tracked farther north in oligotrophic waters, the best fit was a truncated power law with an exponent of 2.02, close to the theoretical optimum for Lévy movement, $\mu_{\mathrm{opt}} \approx 2$ (Supplementary Information, section 2.1, and Supplementary Table 4).

We found that different model fits occurred between different habitat types of the same individual for eight other individuals of five species of predator (bigeye (Thunnus obesus) and yellowfin (Thunnus albacares) tuna, and blue, basking (Cetorhinus maximus) and whale (Rhincodon typus) sharks; see Supplementary Table 4 for model comparisons). For example, a blue shark tracked moving south in the northeast Atlantic, from the highly productive shelf habitat of the western English Channel to the less productive, deep water of the Bay of Biscay, showed switches in the pattern of its vertical movement (Fig. 2a-e). The shark showed diving behaviour in tidal front waters on the shelf (0-200-m depth) that was well fitted to the distribution's tail by an exponential model (Fig. 2a, f, k and Supplementary Table 4). Moving off-shelf into less productive waters (with well-developed thermal stratification) (Fig. $2 \mathrm{~m}, \mathrm{p}, \mathrm{q}$ ), the shark's vertical movements down to $700 \mathrm{~m}$ conformed well to a truncated power law with an exponent of $\mu=2.19$ (Fig. 2b, g, 1), before its diving movements shifted to a pattern better approximated by an exponential fit when in colder, shelf-edge habitat in the southern Bay of Biscay (Fig. 2c, h, m). Returning to warmer, well-stratified but less productive open-ocean habitat (Fig. 2d, e, n, o, q), this shark once again exhibited vertical movements best fitted by truncated power laws with $\mu=1.97$ and 1.99 (Fig. 2i, j).

A bigeye tuna in the central eastern Pacific near the Galapagos Islands switched several times from diving movements best fitted by a truncated power law when in warmer, stratified waters to movements approximated by an exponential model in colder waters of the equatorial convergence front (Supplementary Information, section 2.2).

These results agree with the prediction of the LFF hypothesis that Lévy behaviour should occur in environments where prey is sparsely distributed but that Brownian motion is theoretically optimal where prey is abundant ${ }^{3}$. To test the significance of this with our habitatmapped data, we compared the frequency of sections that conformed to this broad prediction. We assumed that prey in open-ocean habitats with lower primary ${ }^{21}$ and secondary production ${ }^{22}$ was likely to be more sparsely distributed than that in more productive shelf, frontal and convergence-zone habitats, where prey of the predators we tracked is known to be more abundant ${ }^{22-25}$. We used only georeferenced sections yielding best fits where the step-length data spanned at least 1.5 orders of magnitude (range, 1.53-2.27).

For four species of predator (three sharks and ocean sunfish) in the northeast Atlantic that moved between continental-shelf areas with

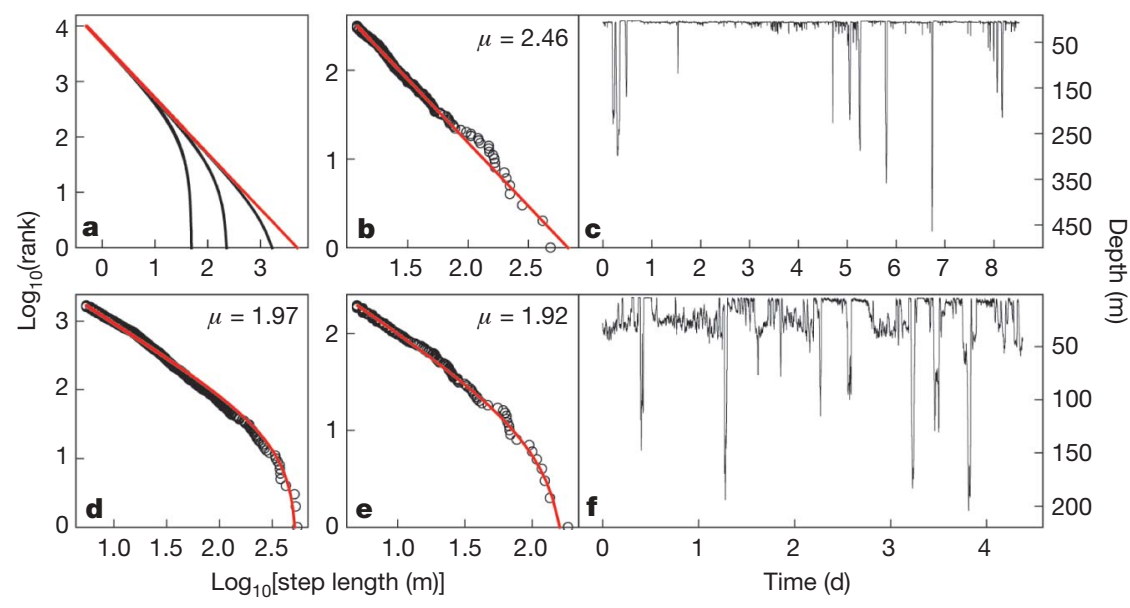

Figure 1 | Examples of good fits to power-law and truncated power-law distributions. a, Synthetic power-law and truncated power-law (Pareto) distributions with upper truncations set to 50, 250, 5,000. b-f, Empirical power-law and truncated power-law fits to dive data from individual blue sharks (Prionace glauca; b, d) and an ocean sunfish (Mola mola, e), together with the diving time series for the individual in $\mathbf{b}$ (over $\sim 8 \mathrm{~d} ; \mathrm{c}$ ) and the individual in $\mathrm{e}$ (over $\sim 4 \mathrm{~d}$; $\mathrm{f}$ ). The red line indicates a synthetic power law in $\mathbf{a}$, a power law in $\mathbf{b}$ and truncated power-law MLE model fits to empirical data in $\mathbf{d}$ and $\mathbf{e}$. 

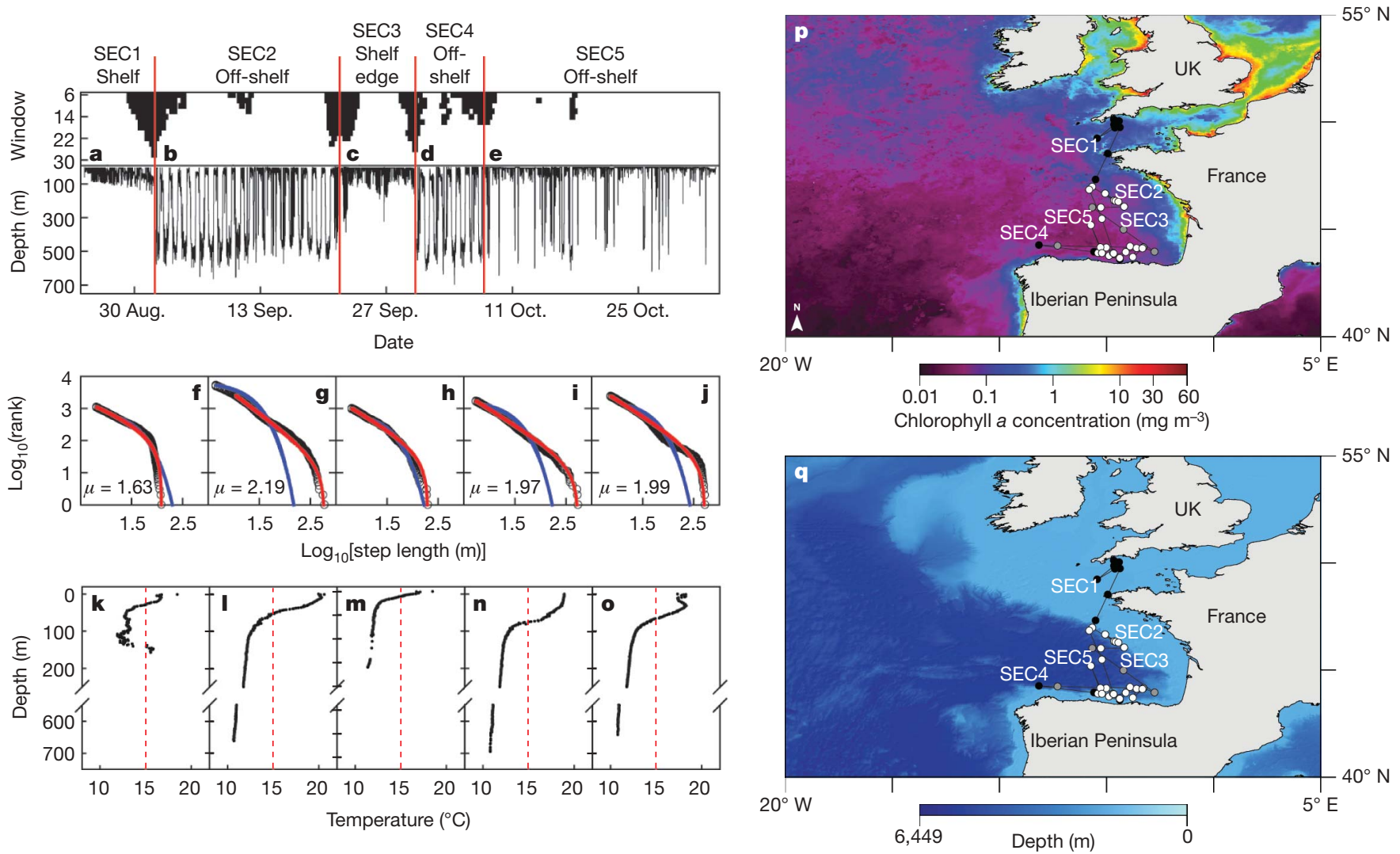

Figure 2 | Behavioural switching between Lévy and Brownian motion in relation to habitat type. a-e, Split moving-window analysis showing significant discontinuities in the dive time series of blue shark 10. Red lines indicate points where the time series was divided into sections (SEC1-SEC5). $\mathbf{f}-\mathbf{j}$, MLE analysis with $\mu$ values for sections best fitting a truncated powerlaw distribution: black circles, observed step lengths; red lines, best-fit truncated power law; blue lines, best-fit exponential distribution. k-o, Depth

high surface zooplankton abundance and open-ocean areas with lower abundance ${ }^{22}$, which provide proxies for prey-abundant and prey-sparse environments, respectively, 14 mapped sections were available. Movement patterns in 12 sections performed as proposed (sparse prey, Lévy behaviour; abundant prey, Brownian motion) (chi-squared test with Yates's correction for continuity: $\chi^{2}=5.78$, $\chi_{0.05,2}^{2}=3.84, P<0.025$; Fig. 3a). This indicates that the frequency of observed movement patterns approximated by a Lévy distribution in less productive areas and by an exponential (Brownian) distribution in more productive waters did not deviate significantly from
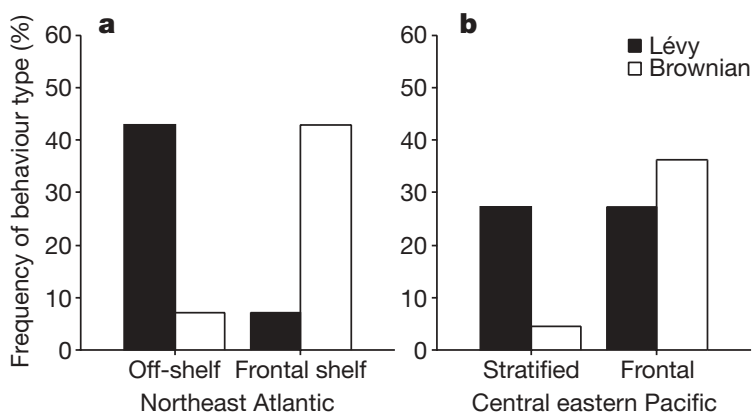

Figure 3 | Spatial occurrence of Lévy and Brownian behaviour types. Frequencies of behaviour types in productive (frontal/shelf) and less productive (off-shelf) habitats in the northeast Atlantic (a), and in productive (frontal) and less productive (stratified) habitats in the central eastern Pacific (b). Tests of two predictions of the LFF hypothesis (Lévy behaviour where prey is sparse; Brownian movement where prey is abundant and not sparsely distributed) were performed on frequency data (not per cent frequency data). See main text for details of the statistical tests. profiles of sea temperature recorded using electronic tags. p, q, Georeferenced track sections of blue shark 10 overlaid on chlorophyll $a$ concentrations ( $\mathbf{p}$ ) and bathymetry $(\mathbf{q})$. Section numbers correspond to those in a-e and different data-point colours correspond to different sections: SEC1, black (higher latitude); SEC2, white (higher latitude); SEC3, grey; SEC4, black (lower latitude); SEC5, white (lower latitude).

theoretical predictions of the LFF hypothesis ${ }^{1,4}$. For bigeye and yellowfin tuna in the central eastern Pacific moving between warm stratified waters and cooler, more productive convergence-front waters (Supplementary Information, sections 2.2 and 2.3) there were 21 sections for analysis. A higher number of sections best fitted by an exponential distribution occurred in convergence-front waters than in stratified waters $\left(\chi^{2}=4.00, \chi_{0.05,2}^{2}=3.84, P<0.05\right.$; Fig. $\left.3 b\right)$. Therefore, the occurrence of Brownian-type behaviour in tuna in the Pacific agrees with predictions of the LFF hypothesis. The number of sections where movements conformed to a truncated power law was the same in convergence-front waters as in stratified waters. We speculate that one reason tuna in the productive convergence zone exhibit Lévy movements characterized by longer vertical steps is that fish prey may become spatially constrained within mesoscale eddy features $^{25}$ that are common in the region and have diameters of between about 50 and $200 \mathrm{~km}$. Thus, even in this productive environment, tuna movement may be optimized by longer vertical reorientations (searching) between eddies because prey hot spots may be patchily distributed across a wide range of scales linked to turbulent eddy formation, size and persistence ${ }^{26}$ (Supplementary Information, sections 2.2 and 2.3).

Our analysis provides the strongest evidence yet for Lévy behaviour in diverse animals ranging across natural landscapes. Furthermore, the movement patterns of some individuals approximated theoretically optimal Lévy searches. It was also evident, however, that Lévy behaviour is not a universal pattern; rather, some individuals use other patterns approximated by normal random processes, sometimes interspersed with Lévy movements. We found that mapping the locations of where Lévy and Brownian movements occurred allowed a preliminary field test of the LFF hypothesis, confirming theoretical predictions. 
Therefore, not only do our results lend strong support to the contention that Lévy flights occur in free-ranging animals, but our observations of pattern switching between Lévy and Brownian-type motion suggest that searching animals adaptively adjust their optimal patterns of movement to different environmental resource distributions. We recognize, however, that our analysis could not detect how the movement patterns arose, that is, whether the patterns identified were an adaptive behaviour or whether observed patterns were an emergent property of the spatial distributions of prey ${ }^{13,17,27}$. Controlled experiments $^{28}$, rather than natural experiments as here, will be needed to progress from asking whether Lévy flights (walks) occur in animals ${ }^{8,9}$ to exploring why they occur and whether animals evolved such that they exploit Lévy flights as an optimal search strategy for life in complex, highly changeable landscapes. Simulations of biological evolution indicate that varying environments posing complex goals can speed up natural selection ${ }^{29}$, which also raises the question of when, if animals have evolved Lévy flight behaviour, did such a strategy first appear among organisms.

\section{METHODS SUMMARY}

Study animals. Animal-attached electronic tags provided time-stamped depth records (tracks) for 55 individuals from 14 species: bigeye thresher shark (Alopias superciliosus), blue shark, shortfin mako shark (Isurus oxyrinchus), porbeagle shark (Lamna nasus), silky shark, oceanic whitetip shark (Carcharhinus longimanus), basking shark, whale shark, bigeye tuna, yellowfin tuna, black marlin (Makaira indica), blue marlin (Makaira nigricans), swordfish (Xiphias gladius) and ocean sunfish.

Track analysis. We used a split moving-window analysis to identify statistically significant discontinuities in depth use and divided tracks into sections that were considered more behaviourally consistent than the whole. Each of the 129 resulting sections was then corrected for sampling artefacts and converted from depths to a series of vertical displacements (move step lengths).

MLE analysis and model selection. For each track section, parameters for power-law, exponential and truncated Pareto distributions were calculated using $\mathrm{MLE}^{16}$. We used plots of ranked move step length, combining empirical and bestfit plots, to reject sections that were a poor fit to all distributions. Log-likelihoods and AIC weights were calculated for the remaining sections and were used to determine which distribution (model) best fitted the data.

\section{Received 1 February; accepted 21 April 2010.}

Published online 9 June 2010.

1. Viswanathan, G. M., Raposo, E. P. \& da Luz, M. G. E. Lévy flights and superdiffusion in the context of biological encounters and random searches. Phys. Life Rev. 5, 133-150 (2008).

2. Viswanathan, G. M. et al. Optimizing the success of random searches. Nature 401, 911-914 (1999).

3. Bartumeus, F. et al. Optimizing the encounter rate in biological interactions: Lévy versus Brownian strategies. Phys. Rev. Lett. 88, 097901 (2002).

4. Bartumeus, F., Da Luz, M. G. E., Viswanathan, G. M. \& Catalan, J. Animal search strategies: a quantitative random-walk analysis. Ecology 86, 3078-3087 (2005).

5. Sims, D. W., Righton, D. \& Pitchford, J. W. Minimising errors in identifying Lévy flight behaviour of organisms. J. Anim. Ecol. 76, 222-229 (2007).

6. Edwards, A. M. et al. Revisiting Lévy flight search patterns of wandering albatrosses, bumblebees and deer. Nature 449, 1044-1048 (2007).

7. Bartumeus, F. Lévy processes in animal movement: an evolutionary hypothesis. Fractals 15, 151-162 (2007).

8. Travis, J. Ecology: do wandering albatrosses care about math? Science 318, 742-743 (2007).

9. Buchanan, M. Ecological modelling: the mathematical mirror to animal nature. Nature 453, 714-716 (2008).

10. Shlesinger, M. F. \& Klafter, J. in On Growth and Form (eds Stanley, H. \& Ostrowsky, N.) 279-283 (Kluwer, 1986).

11. Shlesinger, M. F., Zaslavsky, G. M. \& Klafter, J. Strange kinetics. Nature 363, 31-37 (1993).

12. Viswanathan, G. M. et al. Lévy flight search patterns of wandering albatrosses. Nature 381, 413-415 (1996).

13. Sims, D. W. et al. Scaling laws of marine predator search behaviour. Nature 451, 1098-1102 (2008).

14. White, E. P., Enquist, B. J. \& Green, J. L. On estimating the exponent of power-law frequency distributions. Ecology 89, 905-912 (2008).
15. Edwards, A. M. Using likelihood to test for Lévy flight search patterns and for general power-law distributions in nature. J. Anim. Ecol. 77, 1212-1222 (2008).

16. Clauset, A., Shalizi, C. R. \& Newman, M. E. J. Power-law distributions in empirical data. SIAM Rev. 51, 661-703 (2009).

17. Benhamou, S. How many animals really do the Lévy walk? Ecology 88, 1962-1969 (2007).

18. Bartumeus, F. \& Catalan, J. Optimal search behavior and classic foraging theory. J. Phys. A 42, 434002 (2009).

19. Viswanathan, G. M. et al. Lévy flights in random searches. Physica A 282, 1-12 (2000).

20. Cornelius, J. M. \& Reynolds, J. F. On determining the statistical significance of discontinuities within ordered ecological data. Ecology 72, 2057-2070 (1991).

21. Behrenfeld, M. J. \& Falkowski, P. G. Photosynthetic rates derived from satellitebased chlorophyll concentration. Limnol. Oceanogr. 42, 1-20 (1997).

22. Sims, D. W. et al. Encounter success of free-ranging marine predator movements across a dynamic prey landscape. Proc. R. Soc. B 273, 1195-1201 (2006).

23. Clarke, M. R. \& Stevens, J. D. Cephalopods, blue sharks and migration. J. Mar. Biol. Assoc. UK 54, 949-957 (1974).

24. Moteki, M., Arai, M., Tsuchiya, K. \& Okamoto, H. Composition of piscine prey in the diet of large pelagic fish in the eastern tropical Pacific Ocean. Fish. Sci. 67, 1063-1074 (2001).

25. Zainuddin, M., Kiyofuji, H., Saitoh, K. \& Saitoh, S. I. Using multi-sensor satellite remote sensing and catch data to detect ocean hot spots for albacore (Thunnus alalunga) in the northwestern North Pacific. Deep-Sea Res. I/ 53, 419-431 (2006)

26. Powell, T. M. et al. Spatial scales of current speed and phytoplankton biomass fluctuations in Lake Tahoe. Science 189, 1088-1090 (1975).

27. Boyer, D. et al. Scale-free foraging by primates emerges from their interaction with a complex environment. Proc. R. Soc. B 273, 1743-1750 (2006).

28. Bartumeus, F. et al. Helical Lévy walks: adjusting searching statistics to resource availability in microzooplankton. Proc. Natl Acad. Sci. USA 100, 12771-12775 (2003).

29. Kashtan, N., Noor, E. \& Alon, U. Varying environments can speed up evolution. Proc. Natl Acad. Sci. USA 104, 13711-13716 (2007).

Supplementary Information is linked to the online version of the paper at www.nature.com/nature.

Acknowledgements This research was facilitated through the European Tracking of Predators in the Atlantic programme in the European Census of Marine Life. Funding was provided by the UK Natural Environment Research Council (NERC) Oceans 2025 Strategic Research Programme (Theme 6 Science for Sustainable Marine Resources), the Save Our Seas Foundation, the Leverhulme Trust, the UK Department for Environment Food and Rural Affairs, Fundação para a Ciência e a Tecnologia grant SFRH/BD/21354/2005, the UK Royal Society, the Fisheries Society of the British Isles, the Údarás na Gaeltachta, the Taighde Mara Teo, the Marine Institute (Ireland), the Irish Research Council for Science Engineering and Technology, the Shark Foundation Switzerland, a University of Aberdeen Scholarship and PADI Project Aware. The tuna research of K.M.S. and D.W.F. was made possible through financial contributions by the Japan Fisheries Agency, the US Tuna Foundation and the Tagging of Pacific Pelagics programme in the Census of Marine Life. M.K.M. was funded by Cooperative Agreements NA37RJ0199 and NA67RJ0154 between the National Oceanic and Atmospheric Administration (US Department of Commerce) and the Pelagic Fisheries Research Program (University of Hawaii). The authors or their agencies do not necessarily approve, recommend or endorse any proprietary hardware or software mentioned in this publication. The views expressed herein are those of the authors and do not necessarily reflect the views of their agencies. For field assistance, D.W.S. thanks P. Harris and D. Uren; T.K.D. thanks V. Roantree, M. Norman, M. Lilley and P. F. O'Súilleabháin; J.M.B. thanks G. Adkison, J.-P. Botha, H. Baensch and A. Cumming. D.W.S. and N.E.H. thank A. Clauset and J. Pitchford for help with maximum-likelihood estimation and log-likelihoods, and E. P. White for manuscript comments. This research complied with all animal welfare laws of the countries or sovereign territories in which it was conducted. C.S.J. was supported by a Royal Society of Edinburgh Sabbatical Fellowship, G.C.H. by a Ray Lankester Investigatorship from the Marine Biological Association of the UK (MBA) and D.W.S. by a UK NERC-funded MBA Senior Research Fellowship.

Author Contributions D.W.S. designed the study. N.E.H. and D.W.S. completed data analysis with contributions from N.Q. and J.R.M.D. N.E.H. designed and developed the software for MLE and split moving-window analyses. D.W.S. and N.E.H. wrote the paper and all authors contributed to subsequent drafts. Field data were collected by D.W.S., E.J.S., N.Q., N.G.P., M.K.M., K.M.S., D.W.F., J.M.B., T.K.D., J.D.R.H., G.C.H. and V.J.W.

Author Information Reprints and permissions information is available at www.nature.com/reprints. The authors declare no competing financial interests. Readers are welcome to comment on the online version of this article at www.nature.com/nature. Correspondence and requests for materials should be addressed to D.W.S. (dws@mba.ac.uk). 